\title{
On Quasifree States of the Canonical Commutation Relations (I)
}

\author{
$\mathrm{By}$
}

Huzihiro Araki and Masafumi Shiraishi

\begin{abstract}
A self-dual CCR algebra is defined and arbitrary quasifree state is realized in a Fock type representation of another self-dual CCR algebra of a double size as a preparation for a study of quasi-equivalence of quasifree states.
\end{abstract}

\section{Introduction}

A necessary and sufficient condition for the quasi-equivalence of two quasifree representations of the canonical anticommutation relations (CAR) has been derived in $[11]$ for the gauge invariant case and in $[3]$ for the general case. We shall derive an analogous result for the canonical commutation relations (CCR) in this series of papers.

A quasifree state of $\mathrm{CCR}$ and Bogoliubov automorphisms have been extensively studied $([5] \sim[10],[12],[13])$. We shall use the formulation developped in $[2]$.

In section 2 , we review the formulation in [2]. A self-dual algebra is defined when a linear space $K$, an antilinear involution $\Gamma$ of $K$ and a hermitian form $\gamma$ on $K$ satisfying $\gamma(\Gamma f, \Gamma g)=-\gamma(f, g)^{*}$ are given. In section 3 , we define a quasifree state in terms of a nonnegative hermitian form $S$ on $K$ such that $S(f, g)-S(\Gamma g, \Gamma f)=\gamma(f, g)$. In section 4, the structure of $S$ relative to $(K, \gamma, \Gamma)$ is analyzed.

In section 5 , basic properties of a Fock representation are stated and a result in $[1]$ is quoted. A Fock type representation is defined as a generalization of a Fock representation to the case of degenerate $\gamma$ (i.e. 
the case with nontrivial center). In section 6 , a quasifree state is realized as the restriction of Fock type state of a CCR algebra for $\left(\hat{K}_{S}, \hat{\gamma}_{S}, \hat{\Gamma}_{S}\right)$ where $\hat{K}_{S}$ is about twice as large as $K$.

An application to the quasi-equivalence of quasifree states will be made in a subsequent paper [5].

\section{§ 2. Basic Notions}

Let $K$ be a complex linear space and $\gamma(f, g)$ be a hermitian form for $f, g \in K$. Let $\Gamma$ be an antilinear involution $\left(\Gamma^{2}=1\right)$ satisfying $\gamma(\Gamma f$, $\Gamma g)=-\gamma(g, f) . \quad$ A self-dual CCR algebra $\mathfrak{Y} .(K, \gamma, \Gamma)$ over $(K, \gamma, \Gamma)$ is the quotient of the complex free * algebra generated by $\mathrm{B}(f), f \in K$, its conjugate $\mathrm{B}(f)^{*}, f \in K$ and an identity 1 over (the two-sided $*$ ideal generated by) the following relations:

(1) $\mathrm{B}(f)$ is complex linear in $f$,

(2) $\mathrm{B}(f) * \mathrm{~B}(g)-\mathrm{B}(g) \mathrm{B}(f) *=r(f, g) 1$,

(3) $\mathrm{B}(\Gamma f)^{*}=\mathrm{B}(f)$.

Any one-to-one linear mapping $U$ of $K$ onto $K$ satisfying $r(U f, U g)$ $=\gamma(f, g)$ and $\Gamma U=U \Gamma$ preserves the above relations $(1) \sim(3)$ and hence there exists a unique $*$ automorphism $\tau(U)$ of $\mathfrak{2}(K, \gamma, \Gamma)$ satisfying $\tau(U)$ $\mathrm{B}(f)=\mathrm{B}(U f)$. $\quad U$ and $\tau(U)$ shall be called a Bogoliubov transformation and a Bogoliubov * automorphism.

Any operator $P$ on $K$ satisfying

(1) $P^{2}=P$,

(2) $\gamma(f, P f)>0$, if $P f \neq 0$,

(3) $r(P f, g)=r(f, P g)$,

(4) $Г P \Gamma=1-P$,

is called a basis projection. Such $P$ is linear.

Let $L$ be a complex pre-Hilbert space. A CCR algebra $\mathfrak{A}_{\mathrm{CCR}}(L)$ over $L$ is the quotient of the free $*$ algebra generated by $\left(\mathrm{a}^{\dagger}, f\right),(f, \mathrm{a}), f \in L$ and an identity by (the two-sided $*$ ideal generated by) the following relations:

(1) $\left(\mathrm{a}^{\dagger}, f\right)$ is complex linear in $f$,

(2) $(f, \mathrm{a})=\left(\mathrm{a}^{\dagger}, f\right)^{*}$, 
(3) $\left[(f, \mathrm{a}),\left(\mathrm{a}^{\dagger}, g\right)\right]=(f, g)_{L}$,

$$
\left[\left(\mathrm{a}^{\dagger}, f\right),\left(\mathrm{a}^{\dagger}, g\right)\right]=[(f, \mathrm{a}),(g, \mathrm{a})]=0 .
$$

Let $P$ be a basis projection. Then the mapping $\alpha(P)$ from $\stackrel{\checkmark r}{\checkmark}(K, \gamma$, $\Gamma)$ to $\stackrel{i}{2} \cdot \mathrm{CCR}(P K)$ defined by

$$
\begin{aligned}
& \alpha\left(P,\left(\mathrm{~B}\left(f_{1}\right) \ldots \mathrm{B}\left(f_{n}\right)\right)=\left(\alpha(P) \mathrm{B}\left(f_{1}\right)\right) \ldots\left(\alpha(P) \mathrm{B}\left(f_{n}\right)\right),\right. \\
& \alpha(P) \mathrm{B}(f)=\left(\mathrm{a}^{\dagger}, P f\right)+(P \Gamma f, \mathrm{a})
\end{aligned}
$$

is a $*$ isomorphism of $\stackrel{\Upsilon}{ } \cdot(K, \gamma, \Gamma)$ onto ${ }^{\mathfrak{r}} \cdot \mathrm{CCR}(P K)$.

Let 2 . be a $*$ algebra with an identity. A state $\varphi$ of $\because$ is a complex valued linear functional over $\mathfrak{2}$ satisfying $\varphi(1)=1$ and $\varphi\left(A^{*} A\right) \geqq 0$ for all $A \in \mathcal{Y}$.. Associated with every state $\varphi$, there exists a triplet $\mathfrak{C}_{\varphi}, \pi_{\varphi}$, $\Omega_{\varphi}$ of a Hilbert space, a representation of $\mathfrak{Y}$. by densely defined closable operators $\pi_{\varphi}(A), A \in \mathfrak{Y}$. and a unit vector $\Omega_{\varphi}$, cyclic for $\pi_{\varphi}(\mathfrak{U})$, such that $\varphi(A)=\left(\Omega_{\varphi}, \pi_{\varphi}(A) \Omega_{\varphi}\right), \pi_{\varphi}(A)^{*} \supset \pi_{\varphi}\left(A^{*}\right)$ and the domain of $\pi_{\varphi}(A)$ is $\pi_{\varphi}$ (श) $\Omega_{\varphi}$ •

Let $\operatorname{Re} K$ denote the set of $f \in K$ such that $\Gamma f=f$. It is a real linear space. $f \in \operatorname{Re} K$ if and only if $\mathrm{B}(f)^{*}=\mathrm{B}(f)$.

Let $\varphi$ be a state of $\mathfrak{Y} .(K, \gamma, \Gamma)$ such that $\pi_{\varphi}(\mathrm{B}(f))$ is essentially selfadjoint for all $f \in \operatorname{Re} K$. Let $\left.\mathrm{W}_{\varphi}(f)=\exp i \overline{\pi_{\varphi}(\mathrm{B}(f)}\right), f \in \operatorname{Re} K$. We shall call such state $\varphi$ over $\mathfrak{A}(K, \gamma, \Gamma)$ as a regular state if $\mathrm{W}_{\varphi}(f)$ satisfies the Weyl-Segal relations:

$$
\mathrm{W}_{\varphi}(f) \mathrm{W}_{\varphi}(g)=\mathrm{W}_{\varphi}(f+g) \exp \frac{1}{2} r(g, f) .
$$

Let $\varphi$ be a regular state over $\mathfrak{U}(K, \gamma, \Gamma)$. Let $N_{\varphi}$ be the set of $f \in K$ with $\pi_{\varphi}(\mathrm{B}(f))=0$, which is a linear subset of $K$. Let $\operatorname{Re} N_{\varphi}=N_{\varphi} \cap$ Re $K$. The collection of distances

$$
\mathrm{d}_{\Psi}\left(f, f^{\prime}\right) \equiv \sup _{|t| \leqq 1}\left\|\left\{\mathrm{~W}_{\varphi}(t f)-\mathrm{W}_{\varphi}\left(t f^{\prime}\right)\right\} \Psi\right\|, \Psi \in \mathscr{K}_{\varphi},
$$

defines a vector topology on $\operatorname{Re} K / \operatorname{Re} N_{\varphi}$, which we shall denote by $\tau_{\varphi}$. It also induces a vector topology on $\left(\operatorname{Re} K / \operatorname{Re} N_{\varphi}\right)+i\left(\operatorname{Re} K / \operatorname{Re} N_{\varphi}\right)=K / N_{\varphi}$, which will be denoted again by $\tau_{\varphi}$. The topology induced by one distance $\mathrm{d}_{\Psi}$ for a cyclic $\Psi$ is mutually equivalent and is equivalent to $\tau_{\varphi}[4]$. (The cyclicity here refers to $\mathrm{W}_{\varphi}(f), f \in \operatorname{Re} K$.) 


\section{§3. Quasifree States}

Definition 3.1. A state $\varphi$ on $\mathfrak{2} .(K, \gamma, \Gamma)$ satisfying the following relations is called a quasifree state:

$$
\begin{aligned}
& \varphi\left(\mathrm{B}\left(f_{1}\right) \ldots \mathrm{B}\left(f_{2 n-1}\right)\right)=0 \\
& \varphi\left(\mathrm{B}\left(f_{1}\right) \ldots \mathrm{B}\left(f_{2 n}\right)\right)=\sum \prod_{j=1}^{n} \varphi\left(\mathrm{B}\left(f_{s(j)}\right) \mathrm{B}\left(f_{s(j+n)}\right)\right)
\end{aligned}
$$

where $n=1,2 \ldots$ and the sum is over all permutations $s$ satisfying $s(1)$ $<s(2)<\cdots<s(n), s(j)<s(j+n), j=1, \cdots, n$.

Lemma 3.2. For any state over $\mathfrak{Y} .(K, \gamma, I)$, the hermitian form defined by

$$
\varphi(\mathrm{B}(f) * \mathrm{~B}(g))=S(f, g),
$$

is positive semidefinite (i.e. $S(f, f) \geqq 0$ ) and satisfies

$$
r(g, f)=S(g, f)-S(\Gamma f, \Gamma g) .
$$

Proof. The positivity of $\varphi$ implies the positive semidefiniteness of $S$.

$$
\begin{aligned}
S(\Gamma f, \Gamma g) & =\varphi\left(\mathrm{B}(f) \mathrm{B}(g)^{*}\right)=\varphi\left(\mathrm{B}(g)^{*} \mathrm{~B}(f)\right)-\gamma(g, f) 1 \\
& =S(g, f)-\gamma(g, f) .
\end{aligned}
$$

Lemma 3.3. The hermitian form

$$
(g, f)_{S} \equiv S(g, f)+S(\Gamma f, \Gamma g)
$$

is positive semi-definite and satisfies

$$
\begin{aligned}
& (\Gamma g, \Gamma f)_{s}=(f, g)_{s}, \\
& |\gamma(g, f)|^{2} \leqq(f, f)_{S}(g, g)_{s} .
\end{aligned}
$$

It is positive definite if $\gamma$ is non-degenerate.

Proof. From Lemma 3.2,

$$
S(f, f) \geqq 0, \quad S(\Gamma f, \Gamma f) \geqq 0 .
$$

Hence $(g, f)_{S}$ is positive semidefinite. We also have 


$$
(\Gamma g, \Gamma f)_{S}=S(\Gamma g, \Gamma f)+S(f, g)=(f, g)_{S}
$$

By the Schwarz inequality,

$$
\begin{aligned}
|\gamma(g, f)| & \leqq \\
& \leqq S(g, f)|+| S(\Gamma f, \Gamma g) \mid \\
& \leqq(S(g, g)+S(\Gamma g, \Gamma g))^{\frac{1}{2}}(S(f, f)+S(\Gamma f, \Gamma f))^{\frac{1}{2}} \\
& =(g, g) s^{\frac{1}{2}}(f, f)^{\frac{1}{2}} .
\end{aligned}
$$

If $(f, f)_{S}=0$, we have $\gamma(f, g)=0$ for all $g$. If $\gamma$ is non-degenerate, we have $f=0$. Therefore, $(f, g)_{S}$ is positive definite. - $\quad$ Q. E. D.

Lemma 3.4. The set $N_{S}$ of $f \in K$ satisfying $(f, f)_{S}=0$ is a $\Gamma$. invariant subspace of $K$ such that $S(f, g)=\gamma(f, g)=0$ for any $f \in N_{S}$ and any $g \in K$. If $S$ is related to a state $\varphi$ by (3.3), then $\pi_{\varphi}(\mathrm{B}(f))=0$ is equivalent to $f \in N_{S}$. $\left(N_{S}=N_{\varphi}\right.$ for a regular $\varphi$.)

Proof. From the positive semidefiniteness of $(g, f)_{S}$, it follows that $(g, f)_{S}=0$ for any $g \in K$ whenever $f \in N_{S}$. Hence $N_{S}$ is a subspace of $K$. By (3.6), $N_{S}$ is $\Gamma$-invariant. From (3.7), $\gamma(f, g)=0$ for any $g \in K$ whenever $f \in N_{S}$. This implies that $\mathrm{B}(f), f \in N_{S}$ commutes with all $\mathrm{B}(g), g \in K$. In addition, $0 \leqq S(f, f) \leqq(f, f)_{S}=0$ which implies $\| \pi_{\varphi}(\mathrm{B}(f))$ $\Omega_{\varphi} \|^{2}=S(f, f)=0$ for $f \in N_{S}$. Therefore $f \in N_{S}$ implies $\pi_{\varphi}(\mathrm{B}(f))=0$. Conversely, $\pi_{\varphi}(\mathrm{B}(f))=0$ implies $S(f, f)=\left\|\pi_{\varphi}(\mathrm{B}(f)) \Omega_{\varphi}\right\|^{2}=0, S(\Gamma f, \Gamma f)$ $=\left\|\pi_{\varphi}(\mathrm{B}(f))^{*} \Omega_{\varphi}\right\|^{2}=0$, and hence $(f, f)_{S}=0$.

Lemma 3.5. For any positive semidefinite hermitian $S(g, f)$ on $K \times K$ satisfying (3.4), there exists a unique quasifree state $\varphi_{S}$ satisfying (3.3). Any quasifree state is regular.

Proof. The existence will be seen from Lemma 5.3 and Corollary 6.2. The uniqueness is immediate from (3.1) and (3.2). The regularity will be seen from Corollary 5.6.

Definition 3.6. Let $\mathfrak{S}_{S}, \pi_{S}, \Omega_{S}$ denote the Hilbert space, the repre- 
sentation and the cyclic unit vector canonically associated with the quasifree state $\varphi_{S}$ through the relation

$$
\varphi_{S}(A)=\left(\Omega_{S}, \pi_{S}(A) \Omega_{S}\right), A \in \mathfrak{U} .(K, \gamma, \Gamma) .
$$

If $S$ commutes with a Bogoliubov transformation $U$, then a unitary operator $\mathrm{T}_{S}(U)$ on $\mathfrak{2}_{S}$ is defined by

$$
\mathrm{T}_{S}(U) \pi_{S}(A) \Omega_{S}=\pi_{S}(\tau(U) A) \Omega_{S}
$$

and the continuity. ( $S$ is defined in Lemma 4.2.)

\section{§4. Structure of $(S, K, \gamma, \Gamma)$}

Definition 4.1. $K_{S}$ denotes the completion of $K / N_{S}$ with respect to the positive hermitian form induced on $K / N_{S}$ by $(f, g)_{S} . K / N_{S}$ is identified with a dense subset of $K_{S}$. The Hilbert space topology on $K / N_{S}$ is denoted by $\tau_{S}$.

Lemma 4.2. (1) There exists an antiunitary involution $\Gamma_{S}$ on $K_{S}$ such that $\overline{\Gamma f}=\Gamma_{s} \bar{f}$ for all $f \in K$ where $\bar{f} \equiv f+N_{S} \in K / N_{S}$.

(2) There exists a bounded operator $\gamma_{S}$ on $K_{S}$ such that

$$
\gamma(f, g)=\left(\bar{f}, \gamma_{s} \bar{g}\right)_{s}
$$

for $f, g \in K$. It satisfies

$$
\gamma_{S}^{*}=\gamma_{s}, \Gamma_{s} \gamma_{s} \Gamma_{s}=-\gamma_{s} \quad \text { and } \quad\left\|\gamma_{s}\right\|_{s} \leqq 1 .
$$

(3) There exists a bounded operator $S$ on $K_{S}$ such that

$$
S(f, g)=(\bar{f}, S \bar{g})_{S}
$$

for $f, g \in K$. It satisfies

$$
S^{*}=S, \Gamma_{S} S \Gamma_{S}=1-S, \quad 0 \leqq S \leqq 1,
$$

and

$$
S-\Gamma_{S} S \Gamma_{S}=\gamma_{s} .
$$

Proof. Due to the $\Gamma$-invariance of $N_{s}$ and (3.6), $\bar{\Gamma}_{s} \bar{f}=\overline{\Gamma f}$ defines an antilinear isometric operator on $K / N_{S}$ and hence the closure $\Gamma_{S}$ of 
$\bar{\Gamma}_{S}$ is defined on all vectors in $K_{S}$ and $\left(\Gamma_{S} g, \Gamma_{S} f\right)_{S}=(f, g)_{S}$ for all $f, g \in K_{S}$. Since $\Gamma^{2}=1$, we have $\Gamma_{S}^{2}=1$ and hence $\Gamma_{S}$ is an antiunitary involution on $K_{S}$.

(3.7) and Lemma 3.4 imply the existence of $\gamma_{S}$ satisfying (4.1) and $\left\|\gamma_{s}\right\|_{s} \leqq 1$. Since $\gamma(f, g)$ is hermitian, we have $\gamma_{s}^{*}=\gamma_{s}$. Since $\gamma(\Gamma f$, $\Gamma g)=-\gamma(g, f)$, we have $\Gamma_{s} \gamma_{s} \Gamma_{S}=-\gamma_{s}$.

From the positivity $S(\Gamma f, \Gamma f) \geqq 0$ of $S$, we have $0 \leqq S(f, f) \leqq$ $\|f\|_{S}^{2}$ for $f \in K$. This together with Lemma 3.4 imply the existence of $S$ satisfying (4.3), $S^{*}=S$ and $0 \leqq S \leqq 1$. From (3.5), we have $S+\Gamma_{S} S \Gamma_{S}$ $=1$ and from (3.4), we have (4.5).

Q. E. D.

Definition 4.3. Let $E_{+}, E_{-}$and $E_{0}$ be the spectral projection of $\gamma_{S}$ for $(0,+\infty),(-\infty, 0)$ and $\{0\}$, respectively. Let $K_{ \pm}=E_{ \pm} K_{S}$ and $K_{0}=$ $E_{0} K_{S}$.

Lemma 4.4. $\Gamma_{S} E_{ \pm} \Gamma_{S}=E_{\mp}, \Gamma_{S} E_{0} \Gamma_{S}=E_{0}, \Gamma_{S} K_{ \pm}=K_{\mp}$ and $\Gamma_{S} K_{0}=K_{0}$ Proof. This follows from $\Gamma_{s} \gamma_{s} \Gamma_{s}=-\gamma_{s}$.

Q.E. D.

\section{§ 5. Fock Representations}

Definition 5.1. A quasifree state $\varphi_{S}$ is called a Fock state if the operator $S$ of Lemma 4.2 is a basis projection on $K_{S} . \quad S$ in such a case will be written generally as $P$. The associated representation $\pi_{P}$ is called a Fock representation.

Lemma 5.2. If $P$ is a basis projection of $(K, \gamma, \Gamma)$, then the quasifree state $\varphi_{P}$ of $\mathfrak{Y} \cdot(K, \gamma, \Gamma)$ for $P(f, g)=\gamma(f, P g)$, if it exists, is a Fock state.

Remark. In this case $\gamma$ is automatically non-degenerate and $N_{P}=0$. $P$ originally given on $K$ is a restriction to $K$ of the operator $P$ on $K_{P}$ defined by Lemma 4.2 and we have $r(f, P g)=\left(f, \gamma_{P} P g\right)_{P}=(f, P g)_{P}$ for $f, g \in K$. Therefore the appearance of two $P$ is probably not confusing.

We shall summarize known properties of a Fock state in the following 
3 lemmas.

Lemma 5.3. Let $P$ be a basis projection for $(K, \gamma, \Gamma)$. A state $\varphi$ of $\mathfrak{A}(K, \gamma, \Gamma)$ satisfying

$$
\varphi(\mathrm{B}(f) \mathrm{B}(\Gamma f))=0, \quad f \in P K,
$$

exists, is unique and is a quasifree state $\varphi_{P}$.

Proof. By splitting $\mathrm{B}(f)$ as a sum $\mathrm{B}(P f)+\mathrm{B}((1-P) f)$ and bringing $\mathrm{B}(P f)$ to the left of any other $\mathrm{B}\left((1-P) f^{\prime}\right)$ with a help of the commutation relations, any element $A$ in $\mathfrak{r}(K, \gamma, \Gamma)$ can be written as $A=\sum \mathscr{P}_{i}$ $\mathrm{B}\left(f_{i}\right)+\sum \mathrm{B}\left(g_{j}\right) \mathscr{P}_{j}^{\prime}+\lambda$ where $f_{i} \in(1-P) K, \quad g_{j} \in P K$. Since (5.1) implies $\varphi(Q \mathrm{~B}(f))=\varphi(\mathrm{B}(g) Q)=0$ for $f \in(1-P) K, g \in P K$ and $Q \in \mathfrak{N}(K, \gamma, \Gamma)$ by the Schwarz inequality, we have $\varphi(A)=\lambda$. Hence the uniqueness.

The well known Fock state of $\mathfrak{2}_{\mathrm{CCR}}(P K)$ gives the quasifree state $\varphi_{P}$ through the identification of $\mathfrak{A}_{\mathrm{CCR}}(P K)$ with $\mathfrak{U}(K, \gamma, \Gamma)$ via $\alpha(P)$. $\varphi_{P}$ clearly satisfies (5.1).

Q. E. D.

Lemma 5.4. Let $f \in \operatorname{Re} K$ and $D_{0} \equiv \pi_{P}[\mathscr{N} .(K, \gamma, \Gamma)] \Omega_{P} . \quad D_{0}$ is a dense set of entire analytic vectors of $\mathrm{B}(f)$. The sum

$$
\sum_{n=0}^{\infty} n !^{-1} i^{n} \pi_{P}(\mathrm{~B}(f))^{n}
$$

converges on $D_{0}$. Its closure, denoted by $\mathrm{W}_{P}(f)$, is unitary and satisfies

$$
\begin{aligned}
& \mathrm{W}_{P}\left(f_{1}\right) \mathrm{W}_{P}\left(f_{2}\right)=\mathrm{W}_{P}\left(f_{1}+f_{2}\right) \exp (1 / 2) \gamma\left(f_{2}, f_{1}\right), \\
& \left(\Omega_{P}, \mathrm{~W}_{P}(f) \Omega_{P}\right)=\exp -(1 / 2) \gamma(f, P f) .
\end{aligned}
$$

$f \rightarrow \mathrm{W}_{P}(f)$ is continuous with respect to a norm $\gamma(f, P f)^{1 / 2}$ on $\operatorname{Re} K$ and the strong operator topology on $\mathfrak{C}_{P}$.

Proof. Let $\left(\mathfrak{S}_{P}\right)_{n}$ be the subspace of $\mathfrak{E}_{P}$ generated by $\prod_{j=1}^{n} \pi_{P}\left(\mathrm{~B}\left(g_{j}\right)\right) \Omega_{P}$, $g_{j} \in P K . \quad$ If $\Psi \in \sum_{n=0}^{N}\left(\mathfrak{S}_{P}\right)_{n}$, then

$$
\left\|\pi_{P}(\mathrm{~B}(f)) \Psi\right\| \leqq \sqrt{2}(N+1)^{1 / 2} \gamma(f,(2 P-1) f)^{1 / 2}\|\Psi\| .
$$

[This follows from a well known calculation: Let $\left\{f_{j}\right\}$ be a complete orthonormal basis of $P K$ with $f_{0}=P f$. Then $\Phi=\prod_{\nu=1}^{n} \pi_{P}\left(\mathrm{~B}\left(f_{j_{\nu}}\right)\right) \Omega_{P}(n \leqq N)$ 
is a complete orthonormal basis of $\sum_{n=0}^{N}\left(\mathfrak{Q}_{P}\right)_{n}$, for which $\pi_{P}(\mathrm{~B}(P f)) \Phi$ is also mutually orthogonal and $\left\|\pi_{P}(\mathrm{~B}(P f)) \Phi\right\|=(k+1)^{1 / 2} \gamma(f, P f)^{1 / 2}\|\Phi\|$ where $k$ is the number of $\nu$ with $j_{\nu}=0$. Hence $\left\|\pi_{P}(\mathrm{~B}(P f)) \Psi\right\| \leqq(N+1)^{1 / 2}$ $\gamma(f, P f)^{1 / 2}\|\Psi\|$. A similar calculation with $f_{0}=\Gamma(1-P) f$ leads to $\left.\left\|\pi_{P}(\mathrm{~B}[(1-P) f]) \Psi \vdots \leqq N^{1 / 2} \gamma(f,(P-1) f)^{12}\right\| \Psi \| \cdot\right]$

From (5.5) we have $\lim _{n \rightarrow \infty}\left\|\pi_{P}(\mathrm{~B}(f))^{n} \Psi\right\|^{1 / n} / n=0$ for $\Psi \in D_{0}=\bigcup_{N}\left\{\sum_{n=0}^{N}\right.$ $\left(\left\{_{P}\right)_{n}\right\}$. Hence all such $\Psi$ is an entire analytic vector for $\pi_{P}(\mathrm{~B}(f))$, $f \in \operatorname{Re} K$, (5.2) applied on $\Psi$ converges absolutely, the closure $\bar{\pi}_{P}(\mathrm{~B}(f))$ of $\pi_{P}(\mathrm{~B}(f))$ is selfadjoint, $\mathrm{W}_{P}(f)=\exp i \bar{\pi}_{P}(\mathrm{~B}(f))$, and $\mathrm{W}_{P}(f)$ is unitary. $[14]$. (5.4) follows from $\left(\Omega_{P}, \pi_{P}(\mathrm{~B}(f))^{2 n} \Omega_{P}\right)=(2 n) ! 2^{-n} n !^{-1} \gamma(f, P f)^{n}$.

By the commutation relations, we have

$$
n !^{-1} \mathrm{~B}\left(f_{1}+f_{2}\right)^{n}=\sum_{k+l+2 m=n} k !^{-1} \mathrm{~B}\left(f_{1}\right)^{k} l !^{-1} \mathrm{~B}\left(f_{2}\right)^{l} m !^{-1} 2^{-m} \gamma\left(f_{2}, f_{1}\right)^{m} .
$$

From the previous result and the Schwarz inequality, $\sum k !^{-1} l !^{-1}\left(\mathrm{~B}\left(f_{1}\right)^{k} \Phi\right.$, $\left.\mathrm{B}\left(f_{2}\right)^{l} \Psi\right)$ is absolutely convergent for $\Phi, \Psi \in D_{0}$ and hence we obtain from (5.6) the equality (5.3) for a matrix element between two vectors $\Phi$ and $\Psi$ in a dense set $D_{0}$. Hence (5.3) holds.

From (5.3) and (5.4), we have

$$
\begin{aligned}
& d_{P}\left(f_{1}, f_{2}\right)^{2} \equiv\left\|\left\{\mathrm{W}_{P}\left(f_{1}\right)-\mathrm{W}_{P}\left(f_{2}\right)\right\} \Omega_{P}\right\|^{2} \\
& \quad=2\left\{1-\left(\exp -(1 / 4)\left\|f_{2}-f_{1}\right\|_{P}^{2}\right) \cos (i / 2) \gamma\left(f_{2}, f_{1}\right)\right\}
\end{aligned}
$$

where $\|f\|_{P}^{2} \equiv \gamma(f,[2 P-1] f)$, which is $2 \gamma(f, P f)$ for $f \in \operatorname{Re} K$, and $\gamma\left(f_{2}, f_{1}\right)=\gamma\left(\Gamma f_{2}, \Gamma f_{1}\right)=-\gamma\left(f_{2}, f_{1}\right)^{*}$ is pure imaginary for $f_{1}, f_{2} \in \operatorname{Re} K$. Since $r\left(f_{1}, f_{1}\right)=0$ for $f_{1} \in \operatorname{Re} K$, we have from (3.7)

$$
\left|\gamma\left(f_{2}, f_{1}\right)\right|=\left|\gamma\left(f_{2}-f_{1}, f_{1}\right)\right| \leqq\left\|f_{2}-f_{1}\right\|_{P}\left\|f_{1}\right\|_{P} .
$$

Hence $f \rightarrow \mathrm{W}_{P}(f) \Omega_{P}$ is continuous. By (5.3) and (3.7), this implies the continuity of $f \rightarrow \mathrm{W}_{P}(f) \Psi$ for $\Psi=\mathrm{W}_{P}(g) \Omega_{P}, g \in \operatorname{Re} K$. Since $\pi_{P}(\mathrm{~B}(g))$ $=\lim _{t \rightarrow 0}(i t)^{-1}(\mathrm{~W}(t g)-1)$ on $D_{0}$ for $f \in \operatorname{Re} K$, and since $\mathrm{W}_{P}\left(g_{1}\right) \ldots \mathrm{W}_{P}\left(g_{n}\right)$ $\Omega_{P}$ is a multiple of $\mathrm{W}_{P}\left(\sum g_{j}\right) \Omega_{P}$, finite linear combinations of $\mathrm{W}_{P}(g) \Omega_{P}$, $g \in \operatorname{Re} K$, are dense in $\mathfrak{g}_{P}$. Therefore $f \rightarrow \mathrm{W}_{P}(f)$ is continuous. Q. E. D.

Lemma 5.5. Let $\operatorname{Re} K_{P}$ be the real Hilbert space obtained by the 
completion of $\operatorname{Re} K$ with respect to the inner product $\left(f_{1}, f_{2}\right)_{P}=\gamma\left(f_{1}\right.$, $\left.(2 P-1) f_{2}\right), f_{1}, f_{2} \in \operatorname{Re} K$. If $f=\lim f_{n}, f_{n} \in \operatorname{Re} K$, then $\mathrm{W}_{P}(f) \equiv \lim \mathrm{W}_{P}$ $\left(f_{n}\right)$ exists and does not depend on $\left\{f_{n}\right\}$ for a fixed $f$.

Let $H_{1}$ be a linear subset of $\operatorname{Re} K_{P}$. Denote by $H_{1}^{\perp}$ the set of vectors $f \in \operatorname{Re} K_{P}$ such that $\left(f, \gamma_{P} g\right)_{P}=0$ for all $g \in H_{1}$. Let $\mathrm{R}_{P}\left(H_{1}\right)$ be the von Neumann algebra generated by $\mathrm{W}_{P}(f), f \in H_{1}$. Let $\bar{H}_{1}$ denote the closure of $H_{1}$ in $\operatorname{Re} K_{P}$. Then

(0) $\mathrm{R}_{P}\left(\operatorname{Re} K_{P}\right)$ is irreducible and $\mathrm{R}_{P}(0)$ is trivial,

(i) $\mathrm{R}_{P}\left(H_{1}\right)=\mathrm{R}_{P}\left(\bar{H}_{1}\right)$,

(ii) $\mathrm{R}_{P}\left(H_{1}\right)^{\prime}=\mathrm{R}_{P}\left(H_{1}^{\perp}\right)$,

(iii) $\left(\mathrm{R}_{P}\left(H_{1}\right) \cup \mathrm{R}_{P}\left(H_{2}\right)\right)^{\prime \prime}=\mathrm{R}_{P}\left(H_{1}+H_{2}\right)$,

(iv) $\left(\mathrm{R}_{P}\left(H_{1}\right) \cap \mathrm{R}_{P}\left(H_{2}\right)\right)^{\prime \prime}=\mathrm{R}_{P}\left(\bar{H}_{1} \cap \bar{H}_{2}\right)$,

(v) $\Omega_{P}$ is cyclic for $\mathrm{R}_{P}\left(H_{1}\right)$ if and only if $\bar{P}\left(H_{1}+i H_{1}\right)$ is dense in $\bar{P} K_{P} . \quad\left(\bar{P}\right.$ is the closure of $P$ on $\left.K_{P .}\right)$

(vi) $\Omega_{P}$ is separating for $\mathrm{R}_{P}\left(H_{1}\right)$ if and only if $\bar{P}\left(H_{1}^{\perp}+i H_{1}^{\perp}\right)$ is dense in $\bar{P} K_{P}$,

(vii) $\mathrm{R}_{P}\left(H_{1}\right)$ is a factor if and only if $\bar{H}_{1} \cap H_{1}^{\perp}$ is 0 .

Proof. The existence of the unique limit $\mathrm{W}_{P}(f)$ for $f \in \operatorname{Re} K_{P}$ follows from Lemma 5.4. The von Neumann algebra $\mathrm{R}_{P}\left(H_{1}\right)$ is $\mathrm{R}\left(H_{1} / \mathrm{Re}\right.$ $\left.K_{P}\right)$ in the notation of $[1]$, where $\left(f_{1}, f_{2}\right)_{s}$ and $\gamma\left(f_{1}, f_{2}\right)$ are respectively $\left(f_{1}, f_{2}\right)$ and $\left(f_{1}, \beta f_{2}\right)$. (i) $\sim$ (iv) and (vii) follow from Theorem 1 of $[1]$. (0) and (v) follow from Lemma 5.1 of [1]. (vi) follows from (v) and (ii).

Q. E. D.

Corollary 5.6. A Fock representation is regular and irreducible.

This is due to Lemmas 5.4 and 5.5.

The Fock representation defined above is applicable only for the case of non-degenerate $\gamma$. We now consider its generalization to the case of degenerate $\gamma$.

Definition 5.7. A quasifree state $\varphi_{S}$ in called a Fock type state if $N_{S}=0$ and the spectrum of the operator $S$ in Lemma 4.2 is contained in $\{0,1 / 2,1\}$. The corresponding representation is called a Fock type 
representation.

Lemma 5.8. Let $K, \gamma, \Gamma$ be given. Let $I\left(f_{1}, f_{2}\right)$ be a positive semidefinite hermitian form on $K$ satisfying (3.4), where $S$ is to be replaced by $I I$. Assume that $N_{\Pi}=0$ and the spectrum of the operator $I I$ defined by Lemma 4.2 is contained in $\{0,1 / 2,1\}$. Let $E_{ \pm}, E_{0}$ be defined as in Definition 4.3. Let

$$
\begin{gathered}
\tilde{K}_{I}=K_{I} \oplus E_{0} K_{I I}, \\
\tilde{\Gamma}_{\Pi}(f \oplus g)=\Gamma_{\Pi} f \oplus \Gamma_{\Pi} g, \\
\tilde{\gamma}_{I I}\left(f_{1} \oplus g_{1}, f_{2} \oplus g_{2}\right)=\left(f_{1}, \gamma_{\Pi} f_{2}\right)_{\Pi}+i\left\{\left(g_{1}, f_{2}\right)_{\Pi}-\left(f_{1}, g_{2}\right)_{\Pi}\right\} .
\end{gathered}
$$

Let $\tilde{\mathfrak{Q}} \equiv \mathfrak{P}^{\Upsilon} .\left(\tilde{K}_{I I}, \tilde{\gamma}_{I}, \tilde{\Gamma}_{I I}\right)$ and identify $\mathfrak{\Re} \equiv \mathfrak{2} \cdot(K, \gamma, \Gamma)$ with the subalgebra श्. $\left(K \oplus 0, \tilde{\gamma}_{I}, \tilde{\Gamma}_{I}\right)$ of $\tilde{\mathfrak{A}}$. Let

$$
\begin{gathered}
\tilde{\Pi}(f \oplus g)=\left\{E_{+} f+\left(E_{0} f-i g\right) / 2\right\} \oplus\left\{\left(i E_{0} f+g\right) / 2\right\}, \\
\tilde{\Pi}\left(h_{1}, h_{2}\right)=\tilde{\gamma}_{M}\left(h_{1}, \tilde{\Pi} h_{2}\right) .
\end{gathered}
$$

Then $\varphi_{\tilde{I}}$ is a Fock state of $\tilde{\mathfrak{A}}$ and its restriction to $\mathfrak{A}$ is the Fock type state $\varphi_{\Pi}$.

Proof. $\tilde{\Gamma}_{I}$ is an antiunitary involution of $\tilde{K}_{I I}$ and $\tilde{\gamma}_{I}$ is a hermitian form satisfying $\tilde{\gamma}_{I I}\left(\tilde{\Gamma} h_{1}, \tilde{\Gamma} h_{2}\right)=-\tilde{\gamma}_{I I}\left(h_{1}, h_{2}\right)^{*}$. From (5.12), it follows that $\tilde{\Gamma} \tilde{\Pi} \tilde{\Gamma}+\tilde{\Pi}=1, \tilde{\Pi}^{2}=\tilde{\Pi}$,

$$
\begin{aligned}
\tilde{\gamma}_{\Pi} & \left(f_{1} \oplus g_{1}, \tilde{\Pi}\left(f_{2} \oplus g_{2}\right)\right) \\
& =\left(f_{1}, \Pi f_{2}\right)_{\Pi}+\left(g_{1}, \Pi g_{2}\right)_{I}+i\left\{\left(g_{1}, \Pi f_{2}\right)_{\Pi}-\left(f_{1}, \Pi g_{2}\right)_{\Pi}\right\} \\
& =\tilde{\gamma}_{I I}\left(\tilde{\Pi}\left(f_{1} \oplus g_{1}\right), f_{2} \oplus g_{2}\right),
\end{aligned}
$$

and

$$
\tilde{\gamma}_{\Pi}(f \oplus g, \tilde{\Pi}(f \oplus g)) \geqq 0 .
$$

Therefore $\tilde{I}$ is a basis projection and $\varphi_{\tilde{I}}$ is a Fock state.

$\begin{array}{lll}\text { The restriction of } \varphi_{\tilde{I}} \text { to } \mathfrak{U} \text { is } \varphi_{\Pi} \text { as is seen from (5.14). } & \text { Q. E. D. }\end{array}$

Corollary 5.9. For any $I I$ in Lemma 5.8, the Fock type state $\varphi_{I I}$ 
exists. The commutant $\pi_{\Pi}(\mathfrak{Q})^{\prime}$ is abelian and is generated by $\pi_{I}(\mathrm{~B}(f))$, $f \in E_{0} K_{\Pi \text {. }}$.

Proof. From Lemmas 5.8 and 5.5 (ii), the following computation suffices: If $f \oplus g \in(K \oplus 0)^{\perp}$, then $\left(f, \gamma_{\Pi}\left(1-E_{0}\right) f_{1}\right)_{\Pi}=\left(g, E_{0} f_{1}\right)_{\Pi}=0$ for all $f_{1} \in K$ and hence $f \in E_{0} K_{\Pi}$ and $g=0$.

Q.E. D.

\section{$\S$ 6. A Realization of a Quasifree State on a Fock Type Representation}

Lemma 6.1. (1) Let

$$
\begin{gathered}
K_{S}^{\prime}=K_{S} \oplus K_{S}, \\
\gamma_{S}^{\prime}\left(f_{1} \oplus g_{1}, f_{2} \oplus g_{2}\right)=\left(f_{1}, \gamma_{S} f_{2}\right)_{S}-\left(g_{1}, \gamma_{s} g_{2}\right)_{s}, \\
\Gamma_{S}^{\prime}=\Gamma_{s} \oplus \Gamma_{S} .
\end{gathered}
$$

Then $\Gamma_{S}^{\prime}$ is an antilinear involution and $\gamma_{S}^{\prime}$ is a hermitian form satisfying $\gamma_{S}^{\prime}\left(\Gamma_{S}^{\prime} h_{1}, \Gamma_{S}^{\prime} h_{2}\right)=-\gamma_{S}^{\prime}\left(h_{1}, h_{2}\right)^{*}$. If $N_{S}=N_{S^{\prime}}$ and $\tau_{S}=\tau_{S^{\prime}}$, then there exists a one-to-one linear map $U$ of $K_{S}^{\prime}$ onto $K_{S^{\prime}}^{\prime}$ such that $U h=h$ for $h=(f+$ $\left.N_{S}\right) \oplus\left(g+N_{S}\right), f, g \in K$. It satisfies $U \Gamma_{S}^{\prime}=\Gamma_{S^{\prime}}^{\prime} U$ and $\gamma_{S}^{\prime}\left(h_{1}, h_{2}\right)=\gamma_{S^{\prime}}^{\prime}$ $\left(U h_{1}, U h_{2}\right)$.

(2) Let

$$
\begin{aligned}
& \left(f_{1} \oplus g_{1}, f_{2} \oplus g_{2}\right)_{S}^{\prime}=\left(f_{1}, f_{2}\right)_{S}+\left(g_{1}, g_{2}\right)_{S} \\
& \quad+2\left(f_{1}, S^{1 / 2}(1-S)^{1 / 2} g_{2}\right)_{S} \\
& \quad+2\left(g_{1}, S^{1 / 2}(1-S)^{1 / 2} f_{2}\right)_{S} .
\end{aligned}
$$

Then it is a $\Gamma_{S}^{\prime}$-invariant positive semidefinite form satisfying

$$
\left|\gamma_{S}^{\prime}\left(h_{1}, h_{2}\right)\right| \leqq\left\|h_{1}\right\|_{S}^{\prime}\left\|h_{2}\right\|_{s}^{\prime} .
$$

The kernel $N_{S}^{\prime}$ (i.e. the set of $h$ satisfying $\|h\|_{s}^{\prime}=0$ ) consists of $f \oplus-f$, $f \in E_{0} K_{S}$. If $N_{S}=N_{S^{\prime}}$ and $\tau_{S}=\tau_{S^{\prime}}$, then $N_{S^{\prime}}^{\prime}=U N_{S}^{\prime}$.

(3) (6.4), $\gamma_{S}^{\prime}$ and $\Gamma_{S}^{\prime}$ induce on $K_{S}^{\prime} / N_{S}^{\prime}$ a positive definite inner product $\left(\hat{h}_{1}, \hat{h}_{2}\right)_{S} \equiv\left(h_{1}, h_{2}\right)_{S}^{\prime}$, a hermitian form $\hat{\gamma}_{s}\left(\hat{h}_{1}, \hat{h}_{2}\right) \equiv \gamma_{S}^{\prime}\left(h_{1}, h_{2}\right)$ and an antilinear involution $\hat{\Gamma}_{s} \hat{h} \equiv\left(\Gamma_{s} h\right)^{\wedge}$ satisfying $\left(\hat{\Gamma}_{s} \hat{h}_{1}, \hat{\Gamma}_{s} \hat{h}_{2}\right) \hat{s}=\left(\hat{h}_{2}, \hat{h}_{1}\right) \hat{s}$ and $\hat{\gamma}_{s}\left(\hat{\Gamma}_{S} \hat{h}_{1}, \hat{\Gamma}_{s} \hat{h}_{2}\right)=-\hat{\gamma}_{s}\left(\hat{h}_{2}, \hat{h}_{1}\right)$ where $\hat{h}=h+N_{s}^{\prime} \in K_{S}^{\prime} / N_{s}^{\prime}$. The closure 
of $\hat{\gamma}_{S}$ and $\hat{\Gamma}_{S}$ on the completion $\hat{K}_{S}$ of $K_{S}^{\prime} / N_{S}^{\prime}$, denoted by the same letter, satisfy the same properties. $\hat{\Gamma}_{S}$ is antiunitary and there exists an operator $\hat{\gamma}_{s}$ such that

$$
\begin{gathered}
\hat{\gamma}_{S}\left(h_{1}, h_{2}\right)=\left(h_{1}, \hat{\gamma}_{s} h_{2}\right) \hat{s}, \\
\hat{\gamma}_{S}^{*}=\hat{\gamma}_{s}, \quad \hat{\Gamma}_{s} \hat{\gamma}_{S} \hat{\Gamma}_{S}=-\hat{\gamma}_{S} .
\end{gathered}
$$

If $N_{S}=N_{S^{\prime}}$ and $\tau_{S}=\tau_{S^{\prime}}$, then $U$ of (2) induces a one-to-one linear map of $\hat{K}_{S}$ onto $\hat{K}_{S^{\prime}}$ such that $\hat{U} \hat{\Gamma}_{S}=\hat{\Gamma}_{S^{\prime}} \hat{U}$ and $\hat{\gamma}_{S^{\prime}}\left(\hat{U} \hat{h}_{1}, \hat{U} \hat{h}_{2}\right)=\hat{\gamma}_{s}\left(\hat{h}_{1}, \hat{h}_{2}\right)$.

(4) Let

$$
\Pi_{S}=(1 / 2)\left(1+\hat{\gamma}_{S}\right) \text {. }
$$

Then $\hat{\Gamma}_{S} \Pi_{S} \hat{\Gamma}_{S}=1-\Pi_{S}, \Pi_{S}^{*}=\Pi_{S}$ and the spectrum of $\Pi_{S}$ is contained in $\{0,1 / 2,1\}$.

(5) For $f \in K$, let $[f] \equiv(\bar{f} \oplus 0)+N_{S}^{\prime}$ and identify $K_{S}^{\prime} / N_{S}^{\prime}$ with a dense subset of $\hat{K}_{S}$. Then

$$
\begin{aligned}
& \hat{\gamma}_{s}([f],[g])=\gamma(f, g) . \\
& \left([f], \Pi_{S}[g]\right) \hat{s}=S(f, g) .
\end{aligned}
$$

(6) If $N_{S}=N_{S^{\prime}}$ and $\tau_{S}=\tau_{S^{\prime}}$, then $\tau_{\Pi_{S}}=\tau_{\Pi_{S^{\prime}}}$ and eigenspaces of $\Pi_{S}$ and $\Pi_{S^{\prime}}$ for an eigenvalue $1 / 2$ are mapped by $\hat{U}$.

Proof. (1) The properties of $\Gamma_{S}^{\prime}$ and $\gamma_{S}^{\prime}$ are immediate. Since $K_{S}$ and $K_{S^{\prime}}$ is the completion of $K / N_{S}=K / N_{S^{\prime}}$ with respect to $\tau_{S}=\tau_{S^{\prime}}$, there is a natural identification map $U$ which is linear. If $f_{j}, g_{j} \in K$ and $h_{j}=$ $\left(f_{j}+N_{S}\right) \oplus\left(g_{j}+N_{S}\right)$, then

$$
\begin{aligned}
& r_{S}^{\prime}\left(h_{1}, h_{2}\right)=r\left(f_{1}, f_{2}\right)-r\left(g_{1}, g_{2}\right)=r_{s^{\prime}}^{\prime}\left(h_{1}, h_{2}\right), \\
& \Gamma_{S}^{\prime} h_{1}=\left(\Gamma f_{1}+N_{S}\right) \oplus\left(\Gamma g_{1}+N_{S}\right)=\Gamma_{S^{\prime}}^{\prime} h_{1} .
\end{aligned}
$$

Since such $f_{j}$ and $g_{j}$ are dense in $K_{S}$, these equalities imply $U \Gamma_{S}=\Gamma_{S^{\prime}} U$ and $\gamma_{S}\left(h_{1}, h_{2}\right)=\gamma_{S^{\prime}}\left(U h_{1}, U h_{2}\right)$.

(2) (6.4) is obviously a $\Gamma_{S}^{\prime}$-invariant hermitian form. We have

(6.11) $\quad(f \oplus g, f \oplus g)_{S}^{\prime}=\left\|S^{1 / 2} f+(1-S)^{1 / 2} g\right\|_{S}^{2}+\left\|(1-S)^{1 / 2} f+S^{1 / 2} g\right\|_{S}^{2} \geqq 0$.

We also have

$$
\gamma_{S}^{\prime}\left(f_{1} \oplus g_{1}, f_{2} \oplus g_{2}\right)=\left(S^{1 / 2} f_{1}+(1-S)^{1 / 2} g_{1}, S^{1 / 2} f_{2}+(1-S)^{1 / 2} g_{2}\right)_{S}
$$




$$
-\left((1-S)^{1 / 2} f_{1}+S^{1 / 2} g_{1},(1-S)^{1 / 2} f_{2}+S^{1 / 2} g_{2}\right)_{S}
$$

due to $\gamma_{s}=2 S-1$, which implies

$$
\begin{aligned}
\left|\gamma_{S}^{\prime}\left(f_{1} \oplus g_{1}, f_{2} \oplus g_{2}\right)\right| & \leqq\left\|S^{1 / 2} f_{1}+\left.(1-S)^{1 / 2} g_{1}\right|_{i s}\right\| S^{1 / 2} f_{2}+(1-S)^{1 / 2} g_{2} \|_{S} \\
& +\left\|(1-S)^{1 / 2} f_{1}+S^{1 / 2} g_{1}\right\|_{s}\left\|(1-S)^{12} f_{2}+S^{1 / 2} g_{2}\right\|_{S} \\
& \leqq\left\|f_{1} \oplus g_{1}\right\|_{s}^{\prime}\left\|f_{2} \oplus g_{2}\right\|_{s}^{\prime} .
\end{aligned}
$$

By (6.11), $\|f \oplus g\|_{S}^{\prime}=0$ is equivalent to $(2 S-1) f=0$ and $f+g=0$. Namely $N_{S}$ consists of $f \oplus-f, f \in E_{0} K_{S} . E_{0} K_{S}$ is the set of $f \in K_{S}$ such that $\left(f, \gamma_{S} g\right)_{S}=0$ for all $g \in K_{S}$. If $N_{S}=N_{S^{\prime}}$ and $\tau_{S}=\tau_{S^{\prime}}$, then there is a natural identification of $K_{S}$ with $K_{S^{\prime}}$ which identifies $E_{0} K_{S}$ with $E_{0}^{\prime} K_{S^{\prime}}$ due to $\left(f, \gamma_{s} g\right)_{S}=\gamma(f, g)=\left(f, \gamma_{S^{\prime}} g\right)_{S^{\prime}}$ for $f, g \in K$. ( $E_{0}$ and $E_{0}^{\prime}$ are orthogonal eigenprojections of $S$ and $S^{\prime}$ for an eigenvalue 1/2. Since the orthogonality refers to different inner product, $E_{0}$ and $E_{0}^{\prime}$ need not be the same.) This implies $N_{S^{\prime}}^{\prime}=U N_{S}^{\prime}$.

(3) Immediate from (1) and (2).

(4) Let $\hat{K}_{S}^{+}, \hat{K}_{s}^{-}$and $\hat{K}_{S}^{0}$ be the subspace of $\hat{K}_{S}$ generated by $\left\{S^{1 / 2} f\right.$ $\left.\oplus-(1-S)^{1 / 2} f\right\}^{\wedge},\left\{(1-S)^{1 / 2} f \oplus-S^{1 / 2} f\right\}^{\wedge}$ and $\left\{E_{0} f \oplus E_{0} f\right\}^{\wedge}$, respectively, where $f$ runs over $K_{S}$. It is easily seen that they are mutually orthogonal and altogether generate $\hat{K}_{S}$. For $h_{\sigma}, h_{\sigma}^{\prime} \in \hat{K}_{S}^{\sigma}$ we have $\hat{\gamma}_{S}\left(h_{\sigma}, h_{\sigma^{\prime}}^{\prime}\right)=$ $\sigma \delta_{\sigma \sigma^{\prime}}\left(h_{\sigma}, h_{\sigma^{\prime}}^{\prime}\right) \hat{s}$ where $\sigma=+$, - or 0 . Therefore $\hat{\gamma}_{s} h_{\sigma}=\sigma h_{\sigma}$ and the spectrum of $\Pi_{S}$ is contained in $\{0,1 / 2,1\}$.

(5) Immediate from definitions.

(6) From the proof of (4) and the last part of the proof of (2), it follows that $\hat{K}_{S}^{0}$ for $S$ and $S^{\prime}$ are mapped by $U$ if $N_{S}=N_{S^{\prime}}$ and $\tau_{S}=\tau_{S^{\prime}}$.

The topology $\tau_{\Pi_{S}}$ is the strong topology of $\hat{K}_{S}$. Let $\left(f_{\alpha} \oplus g_{\alpha}\right)^{\wedge}$ be a Cauchy net relative to $\tau_{\Pi_{S}}$ where $f_{\alpha}, g_{\alpha} \in K_{S} . \quad S^{1 / 2} f_{\alpha}+(1-S)^{1 / 2} g_{\alpha} \equiv$ $F_{\alpha}$ and $(1-S)^{1 / 2} f_{\alpha}+S^{1 / 2} g_{\alpha} \equiv G_{\alpha}$ are Cauchy in $K_{S}$. Therefore $f_{\alpha}+g_{\alpha}=$ $\left\{S^{1 / 2}+(1-S)^{1 / 2}\right\}^{-1}\left(F_{\alpha}+G_{\alpha}\right)$ and $(2 S-1)\left(f_{\alpha}-g_{\alpha}\right)=\left\{S^{1 / 2}+(1-S)^{1 / 2}\right\}$ $\left(F_{\alpha}-G_{\alpha}\right)$ are Cauchy. Conversely, if $f_{\alpha}+g_{\alpha}$ and $(2 S-1)\left(f_{\alpha}-g_{\alpha}\right)$ are Cauchy in $K_{S}$, then $F_{\alpha}$ and $G_{\alpha}$ are Cauchy and hence $\left(f_{\alpha} \oplus g_{\alpha}\right)^{\wedge}$ is Cauchy in $\hat{K}_{S}$.

If $N_{S}=N_{S^{\prime}}$ and $\tau_{S}=\tau_{S^{\prime}}$, then the properties of a net $f_{\alpha}$ being Cauchy relative to $\tau_{S}$ and $\tau_{S^{\prime}}$ are the same. Furthermore, $\gamma_{S}=2 S-1$ 
and $\left(f, \gamma_{s} g\right)_{S}=\left(f, \gamma_{S^{\prime}} g\right)_{S^{\prime}}$ imply that $(2 S-1) g_{\alpha}$ is Cauchy relative to $\tau_{S}$ if and only if $\left(2 S^{\prime}-1\right) g_{\alpha}$ is Cauchy relative to $\tau_{S^{\prime}}$ by the duality.

Combining above two sets of arguments, we see that $\left(f_{\alpha} \oplus g_{\alpha}\right)^{\wedge}$ is Cauchy relative to $\tau_{I_{S}}$ if and only if $\left(f_{\alpha} \oplus g_{\alpha}\right)^{\wedge}$ is Cauchy relative to $\tau_{\Pi_{S^{\prime}}}$.

Q.E. D.

Corollary 6.2. The map $f \in K \rightarrow[f] \in K_{S}$ induces a * homomorphism $\alpha_{S}$ of $\mathfrak{U}\left(K, \gamma, I^{\prime}\right)$ into $\mathfrak{U}\left(\hat{K}_{S}, \hat{\gamma}_{S}, \hat{\Gamma}_{S}\right)$. The restriction of a Fock type state $\varphi_{\Pi_{S}}$ of $\mathfrak{Y}\left(\hat{K}_{S}, \hat{\gamma}_{S}, \hat{\Gamma}_{S}\right)$ to $\alpha_{S} \mathfrak{\Re}(K, \gamma, \Gamma)$ gives a quasifree state $\varphi_{S}$ of $\mathfrak{A}(K, \gamma, \Gamma)$ through $\varphi_{\Pi_{S}}\left(\alpha_{S} A\right)=\varphi_{S}(A)$.

This is immediate from Lemma 6.1.

Remark 6.3. It is possible to realize $\varphi_{S}$ directly in a Fock representation in the following manner: Define $K_{S}^{\prime}=K_{S} \oplus K_{S}, \Gamma_{S}^{\prime}=\Gamma_{s} \oplus \Gamma_{S}$,

$$
\begin{aligned}
\gamma_{S}^{\prime \prime}\left(f_{1} \oplus g_{1}, f_{2} \oplus g_{2}\right) & =\left(f_{1}, \gamma_{s} f_{2}\right)_{s}-\left(g_{1}, \gamma_{s} g_{2}\right)_{s} \\
+ & i\left\{\left(g_{1}, E_{0} f_{2}\right)_{s}-\left(f_{1}, E_{0} g_{2}\right)_{s}\right\}
\end{aligned}
$$

and

$$
\begin{aligned}
& \left(f_{1} \oplus g_{1}, f_{2} \oplus g_{2}\right)_{S}^{\prime \prime}=\left(f_{1}, f_{2}\right)_{S}+\left(g_{1}, g_{2}\right)_{S} \\
& \quad+2\left(f_{1},\left(1-E_{0}\right) S^{1 / 2}(1-S)^{1 / 2} g_{2}\right)_{S} \\
& \quad+2\left(g_{1},\left(1-E_{0}\right) S^{1 / 2}(1-S)^{1 / 2} f_{2}\right)_{S} .
\end{aligned}
$$

Then (6.13) is positive definite and

$$
\left|\gamma_{S}^{\prime \prime}\left(h_{1}, h_{2}\right)\right| \leqq\left\|h_{1}\right\|_{s}^{\prime \prime}\left\|h_{2}\right\|_{s}^{\prime \prime}
$$

Let $K_{S}^{\prime \prime}$ be the completion of $K_{S}^{\prime}$ relative to $\|h\|_{S}^{\prime \prime}, \bar{\gamma}_{S}^{\prime \prime}$ and $\Gamma_{S}^{\prime \prime}$ be the closure of $\gamma_{S}^{\prime \prime}$ and $\Gamma_{S}^{\prime}, \bar{\gamma}_{S}^{\prime \prime}\left(h_{1}, h_{2}\right)=\left(h_{1}, \gamma_{S}^{\prime \prime} h_{2}\right)_{S}^{\prime \prime}$ and $P_{S}=\left(\gamma_{S}^{\prime \prime}+1\right) / 2$. Then $P_{S}$ is a basis projection. Let $\alpha_{S}^{\prime \prime}$ be the $*$ homomorphism of $\mathfrak{A}(K, \gamma, \Gamma)$ into $\mathfrak{U}\left(K_{S}^{\prime \prime}, \bar{\gamma}_{S}^{\prime \prime}, \Gamma_{S}^{\prime \prime}\right)$ induced by $f \rightarrow \bar{f} \oplus 0$. Then the restriction of the Fock state $\varphi_{P_{S}}$ of $\mathfrak{U}\left(K_{S}^{\prime \prime}, \bar{\gamma}_{S}^{\prime \prime}, \Gamma_{S}^{\prime \prime}\right)$ to $\alpha_{S}^{\prime \prime 2} \mathfrak{2}(K, \gamma, \Gamma)$ induces the quasifree state $\varphi_{S}$ of $\mathfrak{H}(K, \gamma, \Gamma)$.

This method has a defect that a canonical identification map $U$ can not be defined between $K_{S}^{\prime \prime}, \bar{\gamma}_{S}^{\prime \prime}, \Gamma_{S}^{\prime \prime}$ and $K_{S^{\prime}}^{\prime \prime}, \bar{\gamma}_{S^{\prime}}^{\prime \prime}, \Gamma_{S^{\prime}}^{\prime \prime}$ even if $N_{S}=N_{S^{\prime}}$ and $\tau_{S}=\tau_{S^{\prime}}$, due to the dependence of the operator $E_{0}$ on $S$. 
Lemma 6.4. Let $\varphi_{S}$ be a quasifree state of $\mathfrak{Y}(K, \gamma, \Gamma)$. The induced topology $\tau_{\varphi_{S}}$ on $K$ is the same as $\tau_{S}$ of Definition 4.1.

Proof. Denote $\mathrm{W}_{\varphi_{S}}(f)$ by $\mathrm{W}_{S}(f)$. Since $\Omega_{S}$ is cyclic for $\stackrel{\Upsilon}{\Upsilon}(K, \gamma, \Gamma)$ and $\pi_{s}(A) \Omega_{S}, A \in \mathfrak{S}(K, \gamma, \Gamma)$, is entire for $\overline{\pi_{S}(\mathrm{~B}(f))}=\lim _{t \rightarrow 0} \mathrm{it}^{-1}\left(\mathrm{~W}_{s}(t f)\right.$ $-1), f \in \operatorname{Re} K, \Omega_{S}$ is cyclic for $\mathrm{R}_{S}$.

By [5], it is known that $\tau_{\varphi}$ is a vector topology and is given by one distance $\mathrm{d}_{\Psi}\left(f_{1}, f_{2}\right)$ for a cyclic $\Psi$. Therefore it is enough to show the equivalence of $\|f\|_{S}^{2} \rightarrow 0$ and

$$
d_{s_{S}}(f, 0)=2\left\{1-\exp \left(-\|f\|_{S}^{2} / 4\right)\right\} \rightarrow 0,
$$

where (5.7) is used. This equivalence is obvious.

Q. E. D.

\section{References}

[1] Araki, H., A lattice of von Neumann algebras associated with the quantum theory of a free Bose field, J. Math. Phys. 4 (1963), 1343-1362.

[2] - On the diagonalization of a bilinear Hamiltonian by a Bogoliubov transformation, Publ. RIMS Kyoto Univ. 4 (1968), 387-412.

[3] — On quasifree state of CAR and Bogoliubov automorphisms, Publ. RIMS Kyoto Univ. 6 (1970), 385-442.

[4] Araki, H., and E.J. Woods (to be published).

[5] Araki, H., On quasifree states of the canonical commutation relations (II), this issue.

[6] Dell' Antonio, G.F., Structure of the algebras of some free systems, Comm. Math. Phys. 9 (1968), 81-117.

[7] Manuceau, J., Etude de quelques automorphismes de la $C^{*}$-algèbre du champ de bosons libres, Ann. Inst. Henri Poincaré, 8 (1968), 117-138.

[8] — $\longrightarrow C^{*}$-algèbre de relations de commutation, ibid. 139-161.

[9] Manuceau, J., and A. Verbeure, Quasi-free states of the C.C.R algebra and Bogoliubov transformations, Conm. Math. Phys. 9 (1968), 293-302.

[10] Manuceau, J., F. Rocca and D. Testard, On the product form of quasifree states, Comm. Math. Phys. 12 (1969), 43-57.

[11] Powers, R.T. and E. Størmer, Free states of the canonical auticomnutation relations, Comm. Math. Phys. 16 (1970), 1-33.

[12] Robinson, D.W., The ground state of the Bose gas, Comm. Math. Phys. 1 (1965), 159-171.

[13] Rocca, F., M. Sirugue and D. Testard, On a class of equilibrium states under the Kubo-Martin-Schwinger condition, II, Bosons, Comm. Math. Phys. 19 (1970), 119-141.

[14] Nelson, E., Analytic vectors, Annals of Math. 70 (1959), 572-615. 